\title{
Todos podemos ser creativos. Aportes a la educación
}

\section{We can all be creative. Contributions to education}

DOI: https://doi.org/10.32870/dse.v0i20.590

\author{
Romina Cecilia Elisondo* María \\ Florencia Piga**
}

\begin{abstract}
Resumen
Estudios actuales indican que la creatividad es un potencial que puede ser desplegado en diferentes contextos. El objetivo es analizar, desde la perspectiva de los actores, significados construidos respecto de sus propios procesos creativos, considerando concepciones, actividades y emociones que condicionan la creatividad. El propósito es estudiar los contextos educativos formales y no formales para identificar factores que potencian la creatividad. Presentamos un estudio cualitativo desarrollado en dos etapas, en el primer momento participaron 200 personas adultas (entre 18 y 78 años) de Río Cuarto (Argentina), quienes respondieron un cuestionario sobre creatividad cotidiana. En la segunda etapa, entrevistamos a 20 personas seleccionadas de la muestra inicial. Las entrevistas se centraron en contextos propicios para la creatividad, particularmente en los entornos educativos. Los resultados indican que la creatividad es una capacidad de todas las personas que puede desarrollarse en las más diversas actividades cotidianas. Observamos vinculaciones entre actividades creativas, emociones positivas y satisfacción personal. La creatividad, proceso complejo que incluye componentes subjetivos y contextuales, requiere de ciertas condiciones cognitivas, personales, sociales y culturales para desplegarse en diferentes áreas. Construir contextos educativos creativos implica compromisos y riesgos, además, es necesaria la apertura en las políticas educativas que habiliten intersticios para la innovación.
\end{abstract}

Palabras clave: creatividad cotidiana - autopercepciones - contextos creativos - emociones - innovación educativa.

\begin{abstract}
Current studies indicate that creativity is a potential that can be deployed in different contexts. The aim of this paper is to analyze, from the perspective of the actors, constructed meanings regarding their own creative processes, considering conceptions, activities and emotions that condition creativity. We present a qualitative study developed in two stages. In the first moment, 200 adults (between 18 and 78 years old) from Río Cuarto (Argentina) participated by answering a questionnaire about everyday creativity. In the second stage, we interviewed 20 people selected from the initial sample. The interviews centered on the contexts proposed for creativity, focusing on educational environments. The results indicate that creativi-
\end{abstract}

\footnotetext{
* Doctora en Psicología. Investigadora del Consejo Nacional de Investigaciones Científicas y Técnicas CONICET. Argentina. relisondo@gmail.com **Licenciada en Psicopedagogía por la Universidad Nacional de Río Cuarto. Argentina. florenciapiga_4@hotmail.com
} 
ty is a potential of all people that can be deployed in the most diverse daily activities. We observed links between creative activities, positive emotions and personal satisfaction. Creativity, the complex process that includes subjective and contextual components, requires certain cognitive, personal, social and cultural conditions for deployment in different areas. Creating creative educational contexts involves commitments and risks, and educational policies need to be more open in order to allow for interstices for innovation.

Keywords: everyday creativity - self-perceptions - creative contexts - emotions - educational innovation.

\section{Introducción}

Las teorías actuales indican que la creatividad es un potencial que tienen todas las personas, plausible de ser desarrollado en diferentes actividades artísticas, sociales, culturales y empresariales. Definimos la creatividad como potencialidad vinculado al pensamiento divergente, la generación de productos novedosos y la resolución de problemas de manera alternativa. Runco (2019) conceptualiza la creatividad como un proceso dinámico, personal y parsimonioso. Los componentes principales de este proceso son la originalidad, la efectividad y la autenticidad. Según el autor, el proceso creativo se inicia a partir de interpretaciones personales novedosas sobre las experiencias. Por su parte, los modelos integradores de comprensión de la creatividad explican los procesos creativos como confluencias de factores personales, cognitivos, sociales y culturales (Türst y Grin, 2018). Los paradigmas socioculturales subrayan la importancia de las interacciones entre los creadores, las audiencias, los conocimientos y los objetos sociales. La creatividad se considera como un proceso sociocultural de generación de productos novedosos en determinada cultura (Glaveanu, 2013; 2014). En suma, las definiciones de la creatividad se enfocan en diferentes componentes del proceso: mientras que algunas se centran en aspectos cognitivos y personales, otras destacan la importancia de los condicionantes contextuales. De manera general, podemos definir la creatividad como un constructo complejo que supone relaciones entre componentes personales y sociales para la generación de ideas y productos originales, efectivos y auténticos en diferentes campos de conocimiento y acción.

La vida cotidiana también es un campo donde los potenciales creativos suelen desplegarse para resolver problemas de la más diversa índole. Diferentes estudios empíricos destacan el papel de la autopercepción en el desarrollo de la creatividad (Kaufman, Beghetto y Watson, 2016; Pretz y Kaufman, 2015). Las formas en las que las personas perciben y valoran sus propios potenciales y desempeños parecen condicionar las posibilidades del despliegue de la creatividad. La creatividad es mucho más que un conjunto de recursos cognitivos, es un complejo proceso que integra pensamientos, acciones, interacciones e identidades. La identidad creativa se construye y reconstruye en interacción con otras personas y con base en creencias, represen- 
taciones y autovaloraciones respecto de las propias potencialidades (Beghetto y Karwowski, 2017; Glaveanu, 2017).

Organismos internacionales como la UNESCO $(2014 ; 2015)$ subrayan la importancia de la creatividad como tópico que atraviesa agendas referidas a patrimonio, género y desarrollo social. La creatividad, en tanto capacidad para resolver problemas, es una herramienta para la transformación social y la búsqueda de alternativas a problemáticas actuales. Asimismo, en las políticas de la UNESCO se destacan acciones orientadas al desarrollo de industrias creativas, la promoción de prácticas culturales diversas y la inclusión a partir del arte y las economías alternativas.

La creatividad se articula permanentemente con la cultura y el desarrollo social en tanto forma de generar alternativas y nuevos significados en las comunidades:

Para aprender a vivir con la incertidumbre, a mi juicio, es necesario cultivar la creatividad humana $-y$ recuperar el sentido original de "cultura" en tanto que acción de cultivar - para que los individuos, las comunidades y las sociedades puedan adaptarse con imaginación y capacidad de innovación a la nueva era global. La Comisión de Cultura y Desarrollo, en su informe Nuestra Diversidad Creativa, no se refiere exclusivamente a la creatividad necesaria para la producción individual de un objeto al que se atribuye un valor estético (un objeto de arte) sino a la creatividad necesaria para inventar nuevas formas de organizarse en sociedad y crear nuevos sentidos (Arizpe, 2001:31).

Considerando la relevancia social y teórica de la creatividad en el contexto actual, nos proponemos en el presente estudio analizar, desde la perspectiva de los actores, significados construidos respecto de sus propios procesos creativos. Nos interesa analizar concepciones, actividades, contextos y emociones que condicionan estos procesos, recuperando las voces de los actores en relación con sus potencialidades y acciones creativas. Es nuestro propósito enfocarnos en los contextos educativos formales y no formales para identificar condiciones que potencian la creatividad en estos entornos.

Presentamos un estudio cualitativo fenomenológico desarrollado en dos etapas, en el primer momento participaron 200 personas adultas residentes en la ciudad de Río Cuarto (Argentina) cuyas edades oscilan entre 18 y 78 años. En esta etapa, las personas respondieron un cuestionario sobre concepciones de la creatividad y actividades en las que se consideraban creativos. En la segunda etapa, se seleccionaron 20 casos de la muestra inicial; estas personas fueron entrevistadas. Las entrevistas semiestructuradas se centraron en actividades y contextos propicios para la creatividad, particularmente en los entornos educativos y sus condicionantes. Los análisis realizados muestran que la creatividad es considerada por los participantes como una potencialidad que puede desarrollarse en diversas actividades de la vida diaria, generando emociones positivas y satisfacción personal. El campo educativo aparece como el contexto 
donde la creatividad puede desarrollarse; entre los factores promotores, los participantes señalan: aprendizaje experiencial, autonomía, búsqueda de alternativas y resolución de problemas diversos.

En síntesis, esperamos contribuir a la comprensión de la creatividad como proceso complejo que puede desarrollarse en diversos campos, destacando el papel de los contextos educativos. Es nuestra intención aportar evidencias, desde las voces de los actores, respecto de las acciones que favorecen procesos creativos en la educación.

\section{Contexto conceptual}

En este apartado se consideran tres aspectos principales: el campo actual de investigación de la creatividad, la identidad creativa, y los procesos creativos en entornos educativos.

El campo actual de la investigación sobre creatividad se caracteriza por la multiplicidad de definiciones, enfoques y metodologías. Múltiples preguntas, problemas y desafíos estructuran un complejo de posibles líneas en la investigación de la creatividad. Según Türst y Grin (2018), el campo de la investigación de la creatividad se basa en cuatro preguntas principales: la primera, referida a los niveles de creatividad (creatividad con mayúsculas, creatividad con minúsculas, pro-creatividad y minicreatividad); la segunda, a las distinciones entre potencial y logro; la tercera, al debate entre el dominio general y específico; y la última, a la diferencia entre persona, proceso, producto y entorno. Los investigadores enfocan sus estudios de diferentes maneras, considerando las perspectivas que asumen para responder estas preguntas principales.

Nuestra investigación se centra en el nivel de creatividad cotidiana, específicamente en las percepciones de la personas respecto de sus propios potenciales y desempeños creativos. Según Richards (2010), la creatividad cotidiana se define en términos de originalidad humana en el trabajo y el ocio, se manifiesta en diversas actividades de la vida cotidiana y es fundamental para la supervivencia. Las personas pueden ser creativas en diferentes acciones cotidianas. La creatividad es posible en diferentes dominios y contextos. Permanentemente, las personas resuelven problemas cotidianos de forma novedosa y crean productos originales.

Entendemos que la creatividad es siempre un proceso social que incluye a otras personas (Elisondo, 2016) y que se desarrolla en interacción permanente con un contexto cultural más amplio, en el que se comparten prácticas y significados (Glaveanu, 2014; 2013). Nuestro estudio reconoce interacciones complejas entre personas, procesos, productos y entornos en el despliegue de la creatividad. Como sostiene Glaveanu (2013), los procesos creativos implican interrelaciones entre actores, acciones, artefactos, audiencias y contextos. Según este mismo autor (2018), la creatividad no es una construcción o un fenómeno unitario, es un concepto aplicado a una variedad de acciones o actividades humanas que conducen a resultados apreciados como novedosos, originales, valiosos o significativos. El autor invita a ser sensibles a las múltiples crea-

DiáloQos sobre Educación año 11 | número 20 | enero-junio 2020 | ISSN 2007-2171 
tividades, a cultivar la diversidad y a ofrecer las herramientas y los apoyos necesarios para que las personas desarrollen sus propios estilos creativos y formas de expresión.

El presente estudio se refiere al análisis de autopercepciones construidas por los participantes. En las teorías actuales de la creatividad, las autopercepciones y autovaloraciones son un tema de relevancia. Las formas en que las personas perciben sus propias capacidades y logros creativos es un factor a tener en cuenta en los procesos de desarrollo de la creatividad en diversos contextos. Estas construcciones personales condicionan los procesos creativos, además, son dinámicas e intersubjetivas, se construyen y reconstruyen permanentemente en interacción con otras personas. Varias investigaciones han observado efectos de las autopercepciones en la creatividad (Kaufman, Beghetto y Watson, 2016; Pretz y Kaufman, 2015). Es decir, estas construcciones subjetivas inciden en los desempeños creativos de las personas condicionando sus logros y posibilidades.

Coincidimos con Glaveanu (2017) en la importancia de investigar el Ser creativo y los elementos que lo constituyen. El análisis de las creencias construidas por las personas con respecto a sus propias habilidades creativas es relevante para comprender los procesos de despliegue de la creatividad en contextos cotidianos.

En conjunto, estas tres creencias propias influyen en la identidad creativa de una persona (Cl), evaluaciones generales (o similares a rasgos) de habilidades creativas (CSC), autoevaluación y evaluaciones de tareas de la viabilidad y adecuación de la participación creativa con situaciones particulares (CMC) y una confianza más específica (o similar a un estado) en la realización creativa de tareas particulares, en momentos particulares, en particular contextos (CSE). (Beghetto y Karwowski, 2017: 9).

La identidad se construye en la compleja red de representaciones con respecto a las capacidades y los logros propios. Este marco está formado por el Yo en interacción con los demás, integrando experiencias pasadas, actividades presentes y proyectos futuros.

En el estudio también interesa analizar especialmente los contextos educativos y cuáles son las condiciones, según la perspectiva de los participantes, que favorecen el desarrollo de la creatividad. Tal como sostienen los especialistas (Gajda, Beghetto y Karwowski, 2017; Glaveanu, 2018; Richardson y Mishra, 2018), la creatividad es un proceso indispensable en los aprendizajes que habilita la construcción de nuevos conocimientos y relaciones entre saberes. La creatividad es posible en diferentes áreas curriculares, siendo las actividades interdisciplinarias las que parecen ser más propicias. Se destaca la importancia de las concepciones de los docentes en la potenciación de la creatividad (Kettler, Lamb, Willerson y Mullet, 2018; Mullet, Willerson, Lamb y Kettler, 2016). Glaveanu (2018) considera relevante que los educadores construyan concepciones amplias de la creatividad que les permitan reconocer las diversas situaciones donde las 
personas pueden ser creativas. Además, es necesario que los docentes brinden los andamios necesarios para estimular la creatividad en actividades áulicas y extraáulicas.

Tal como define Beghetto (2017), la enseñanza creativa refiere a la creación de contextos donde los protagonistas toman riesgos, aprenden de los errores, trabajan en cierta incertidumbre de manera flexible, explorando nuevas ideas, posiciones y experiencias. Construir contextos creativos de enseñanza y aprendizaje supone desarrollar modelos de actuación propios del pensamiento creativo (apertura, flexibilidad, originalidad, divergencia).

Gajda, Beghetto y Karwowski (2017) plantean un conjunto de comportamientos que resultan propicios para el aprendizaje creativo:

- establecer metas de aprendizaje enfocadas en la mejora,

- brindar oportunidades para que los estudiantes usen su imaginación mientras aprenden,

- alentar a los estudiantes a tomar riesgos razonables y actuar de forma independiente,

- enseñar con un enfoque que propicie el juego,

- brindar oportunidades de elección y descubrimiento,

- fomentar el pensamiento flexible y la confianza en las ideas de los estudiantes,

- promover el compromiso de los estudiantes en los aprendizajes individuales y cooperativos.

El clima de la clase juega un papel importante en la creatividad. Richardson y Mishra (2018) enfatizan la importancia de crear una atmósfera de cooperación en la que los estudiantes puedan tomar riesgos, cometer errores y plantear ideas alternativas. Asimismo, es necesario construir en el aula una comunidad respetuosa, atenta, tolerante con las diferencias y abierta a la comunicación.

Hemos presentado algunos planteos teóricos que resultan de relevancia para el estudio desarrollado. En síntesis, comprendemos la creatividad como una potencialidad de todas las personas que puede desarrollarse en diferentes contextos y situaciones. Destacamos el papel de las autopercepciones y autovaloraciones en la construcción de la identidad creativa y el desarrollo de la creatividad. Por último, presentamos algunos posicionamientos teóricos actuales respecto de los procesos creativos en entornos educativos, explicitando decisiones y acciones que resultan propicias para la creatividad en el aprendizaje y la enseñanza. La creatividad es un constructo complejo, es mucho más que procesos cognitivos, es una trama de representaciones, identidades, interacciones y acciones en contextos particulares. En definitiva, la creatividad es un proceso sociocultural, que se construye a partir de relaciones entre el Yo, los otros, los artefactos, las acciones, las interacciones y las mediaciones del lenguaje. 


\section{Método}

Desarrollamos un estudio cualitativo fenomenológico en dos etapas, en la primera, 200 personas respondieron un cuestionario abierto sobre creatividad cotidiana, en la segunda, seleccionamos y entrevistamos a 20 personas de la muestra inicial. Se trata de un estudio fenomenológico porque se intenta captar los objetos de estudio tal como son percibidos por los participantes (Creswell y Poth, 2007). Es decir, se analizan sentidos y significados construidos respecto de la creatividad y los factores que la condicionan. Para la construcción de las categorías de análisis se triangularon datos recolectados en las dos etapas de la investigación.

En la etapa 1 participaron estudiantes universitarios, docentes y grupos diversos de adultos de diferentes edades que residen en la ciudad de Río Cuarto. 61.5\% de los participantes son mujeres. Las edades oscilan entre los 18 y 78 años, aunque la mayor cantidad se encuentran entre los 18 y 35 años. Utilizamos un muestreo de tipo intencional y por conveniencia, incluimos en la muestra personas que cumplieran criterios de diversidad en cuanto sexo y edad. En esta etapa de la investigación utilizamos un cuestionario abierto para la recolección de datos. El instrumento incluye ítems referidos a concepciones de la creatividad, actividades creativas y contextos donde dichas actividades son desarrolladas por los participantes.

En la etapa 2, luego del análisis de los datos recogidos mediante los cuestionarios, seleccionamos a 20 personas para la realización de entrevistas semiestructuradas. En esta etapa utilizamos un muestreo basado en el siguiente criterio: incluir en la muestra personas que se desempañaran en diferentes actividades académicas y laborales, procurando incluir varios docentes y estudiantes considerando el interés por analizar condicionantes de la creatividad en entornos educativos. Presentamos a continuación datos sociodemográficos de los participantes de la segunda etapa: Abigail (24 años, psicomotricista y acompañante terapéutico, trabaja en un dispensario y en un centro de rehabilitación); Carina (21 años, trabaja en limpieza y atención al público); Aldana (21 años, estudiante avanzada de Educación Física, trabaja en un club); Luis (31 años, estudiante avanzado del profesorado de Psicología, trabaja en la secretaría de un colegio secundario); Giuliana (24 años, profesora de Educación Física); Alejandra (33 años, profesora de bellas artes); Laura (59 años, jubilada docente); Bárbara (26 años, abogada, trabajos administrativos y jurídicos en una financiera); Santiago (25 años, profesor de Educación Física); Tania (41 años, profesora de Historia); María Cecilia (33 años, secundario, comerciante y ama de casa); Santiago (27 años, comunicador social); Viviana (52 años, maestra jardinera, vicedirectora); Laura (26 años estudiante avanzada de Ciencias Políticas); Miguel (24 años, estudiante de Psicología, profesor de Teatro); Andrés ( 25 años, estudiante de comunicación social, trabaja en una agencia de comunicación); Francesco (22 años, estudiante de Ingeniería Agronómica); Jorge (57 años, docencia universitaria e investigación); Valentina (34 años, licenciada en relaciones públicas e institucionales, realiza trabajos administrativos en una empresa familiar); y Carina (46 años, docente de primaria).

En esta segunda etapa utilizamos entrevistas semiestructuradas como instrumentos de re- 
colección de datos. El guion de la entrevista se construyó con base en los siguientes ítems: percepciones sobre creatividad y contextos educativos, laborales y de tiempo libre, condicionantes que inciden en los procesos creativos y emociones emergentes en dichos procesos.

Los datos fueron codificados y analizados mediante el método de comparaciones constantes (Soneira, 2006). A partir de las codificaciones y las comparaciones se construyeron categorías y teorías emergentes de los datos. Los resultados se interpretaron a la luz de planteos teóricos y estudios actuales de la creatividad.

Desarrollamos el proceso de investigación conforme a pautas éticas establecidas para investigaciones en Ciencias Sociales. Solicitamos el consentimiento informado a los participantes, los datos fueron tratados de manera confidencial preservando el anonimato.

\section{Desarrollo y resultados}

A partir de la triangulación y el análisis cualitativo de los datos construimos cuatro categorías: concepciones de creatividad, actividades creativas, emociones en el proceso creativo y creatividad en contextos educativos. A continuación presentamos las categorías, algunas evidencias empíricas que las ilustran y los correspondientes análisis teóricos. En los análisis se articulan evidencias recolectadas a partir de los cuestionarios y las entrevistas; se presentan de esta manera considerando los procesos de triangulación realizados.

\section{Concepciones de creatividad}

En su gran mayoría, los entrevistados definieron la creatividad como una habilidad o capacidad de generar algo nuevo. Estas concepciones destacan uno de los componentes principales de la creatividad: la originalidad, es decir la capacidad de generar ideas o productos novedosos, únicos e inusuales (Runco y Jaeger, 2012). Las expresiones de los participantes también destacan a la imaginación como un componente clave en los momentos iniciales de la creatividad. La imaginación se refiere a representaciones mentales de cosas o ideas que no se presentan inmediatamente a los sentidos. Las imágenes mentales tienen un papel importante en la creatividad, en tanto generan alternativas y posibilidades, tal como destacan los estudios cognitivos de los procesos creativos (Ward, 2007).

Creatividad para mí es la capacidad de poder crear cosas desde nuestra imaginación (Osvaldo, 45 años).

Es una habilidad de una persona que tiene para imaginar, crear o innovar algo saliendo de lo común (Milagros, 20 años).

La creatividad es tener la capacidad de crear cosas a través de la imaginación, la creatividad nace desde el interior de uno (Evelin, 22 años).

Algunos participantes definen la creatividad como herramienta para resolver problemas 
diversos. Estas definiciones se vinculan con los planteos de la UNESCO (1994), que entienden la creatividad como capacidad de resolver problema y transformar la realidad. Algunos especialistas han señalado complejas relaciones entre creatividad y problemas: mientras que algunos sostienen que el pensamiento divergente se asocia a la capacidad de resolver problemas de manera creativa, otros consideran que formular problemas es lo específico de la creatividad (Kozbelt, Begheto y Runco, 2010).

Es la manera de resolver las cosas, son las herramientas, experiencias y ese tipo de cuestiones que ayudan a resolver distintos tipos de problemas, desafíos o cualquier cosa que se nos presente (Maximiliano, 29 años).

En las voces de los participantes también observamos concepciones que definen la creatividad como libertad para expresar ideas, emociones y deseos mediante la creación de diversos productos y proyectos. En este sentido, destacamos la importancia de los procesos creativos como oportunidades para la expresión personal, la comunicación de emociones y la autorrealización. Estudios anteriores subrayan la relevancia de la expresión creativa en el bienestar y la salud de las personas (Gandolfo y Marty, 2010; McCabe, 2015; Pollanen, 2015).

La creatividad es una forma de expresar ideas, emociones o pensamientos con originalidad (Daniela, 32 años).

Es la capacidad de poder expresar lo que imaginamos, y también cumplirlo (Enrique, 55 años).

La creatividad es una forma de expresarse. Permite ampliar la mente y ver las cosas de diferentes formas (Sebastián, 30 años).

Las concepciones de los participantes se vinculan con diferentes aspectos de los procesos creativos; mientras que algunas expresiones se refieren a los componentes cognitivos como la imaginación y la originalidad, otras destacan las potencialidades subjetivas de la creación como medio de expresión personal. Las voces de los participantes también vinculan la creatividad con la resolución de problemas diversos. Las concepciones de los participantes se relacionan con teorías actuales de la creatividad que la definen como potencialidades de todas las personas para resolver situaciones diversas y crear productos novedosos.

La creatividad no es sólo una cosa de artistas, diseñadores industriales, grandes empresarios, inventores, publicistas o políticos, que también es de todos. En cualquier diminuto puesto de trabajo, en la escuela, en los pequeños y grandes negocios, en la concejalía o la consejería, poder contar con la persona 
creativa en el puesto adecuado supone la posibilidad de subsistir en tiempos cambiantes, de crecer, de optimizar los recursos, de alcanzar la excelencia. En la vida cotidiana, en el ocio, en las relaciones humanas, en la pareja, en los propios planteamientos vitales, haber logrado desarrollar la creatividad y permitir que fluya confiadamente, puede marcar la diferencia entre el hastío y la alegría, entre la depresión y la autorrealización (Corbalán, 2008: 16).

Desde nuestro punto de vista, estas concepciones son valiosas en tanto muestran amplitud de campos posibles y reconocen potencialidades en todas las personas. Estas perspectivas resultan propicias para la educación, ya que si consideramos que la creatividad es una potencialidad que puede desarrollarse en diferentes campos, el papel de los contextos educativos puede ser decisivo. Los contextos de enseñanza y aprendizaje pueden ofrecer múltiples alternativas, actividades y conocimientos que promuevan la creatividad en diversos campos disciplinares y acciones cotidianas.

\section{Actividades creativas}

Los entrevistados mencionan una amplia variedad de actividades en las que se consideran creativos. Estas expresiones se articulan con las concepciones analizadas en la categoría anterior; en tanto se considera que la creatividad es una potencialidad de las personas, múltiples actividades aparecen como propicias para desarrollar estas capacidades. El arte, las artesanías, el diseño, la cocina, la estética personal y el cuidado del hogar aparecen como campos cotidianos donde las personas desarrollan la creatividad.

En actividades como dibujar, pintar, hacer trabajos prácticos, en tareas del hogar (Agustina, 23 años).

Soy creativo en hacer plantas, diseñar parques y jardines, de chico era creativo en juegos (Carlos, 38 años).

Tejido, decoración de salón (a veces me contratan para eso), arreglo floral (Marcela, 46 años).

En teatro, y siempre me gustó interpretar los papeles que me tocaban. Los creaba yo, los hacía yo, los improvisaba y eso le gustaba a la directora. A veces le escribía el libreto a los demás, tengo mucha espontaneidad e imaginación (María, 34 años).

En los instrumentos, todo lo relacionado con la música (María Luz, 18 años).

Siempre fui muy creativa, de reciclar y volver a utilizar las cosas, la mayoría del tiempo me dedico a re-

Diólo@os

sobre Educación año 11 | número 20 | enero-junio 2020 | ISSN 2007-2171 
ciclar cosas para mi patio y a pintar masetas, una de mis actividades preferidas es la jardinería (Carmen, 51 años).

Haciendo manualidades, como pintar (Sofía, 23 años).

Algunas personas destacan ser creativos ante situaciones problemáticas personales y de otras personas. Ayudar a otros, adaptase a nuevas situaciones y espacios, afrontar problemas, también demandan procesos creativos tal como lo sostienen los participantes. El cuidado de otras personas también es una actividad en la que algunos participantes se definen como creativos.

Quizás soy una persona creativa para situaciones en las que las personas necesitan de una ayuda, situaciones problemáticas, por ejemplo (Milena, 20 años).

Soy creativa al momento de pasar una mala situación económica (Verónica, 32 años).

En adaptarme a nuevos espacios, conocer gente nueva... (Sebastián, 30 años).

En la vida, porque tenes que ser creativo en todo, cuando le enseñas una canción a tu hijo, cuando buscas la forma de que tu hijo entienda la cuenta, yo creo que, en la vida, en todo (Paola, 39 años).

En cuidar a mi hermana, lograr que no se aburra y hacer actividades para mi casa (Macarena, 20 años).

Muchos participantes se refieren a actividades en contextos educativos como campos propicios para la creatividad. Algunos recuerdan acciones y proyectos particulares de los que participamos como estudiantes, y otros señalan trabajos concretos como docentes. Destacamos la importancia de estas expresiones que indican que la escuela es un espacio donde se desarrollan actividades creativas, tanto en procesos de aprendizaje como de enseñanza. Contradiciendo planteos que indican que las instituciones educativas obstaculizan la creatividad, las voces de los participantes recuperan diversas experiencias creativas en escuelas, universidades y espacios educativos no formales.

En los ensayos en mi escuela, hacemos muchos juegos y me gusta inventar juegos. En literatura me gusta hacer cuentos, poemas, rimas (Marien, 18 años).

Soy creativo en diseñar, por ejemplo, en el secundario nos hacían hacer proyectos, en el industrial, y me explayaba (Agustín, 24 años).

Siento que fui creativa cuando tuve que componer canciones en una actividad que tuve que llevar a 
cabo en la escuela secundaria... (Milagros, 20 años).

En trabajos de arte del colegio (Celeste, 18 años).

Siempre fui muy creativa a la hora de resolver problemas, o situaciones, como por ejemplo matemáticos o geométricos (Silvana, 22 años).

En el nivel educativo, académico, lo que fueron los trabajos prácticos, las exposiciones, y en el ámbito laboral a la hora de planificar las clases, los métodos, las técnicas para enseñar inglés y Lengua... (Andrea, 26 años).

En mi trabajo, en el dictado de clases y forma de enseñanza (Bruno, 29 años).

En hacer para los niños cuando era docente, buscar actividades divertidas, y que se relacionaran con la vida diaria en la cual ellos se iban a insertar (Marta, 64 años).

En mi trabajo, porque hace muchos años que trabajo de seño, y eso implica tener que ordenarme, y crear e imaginar actividades y cosas para todas las semanas. Que a mis alumnos le guste e interese mis clases (Fabiana, 40 años).

Algunos participantes sostienen que son creativos en actividades físicas, deportivas y referidas a la expresión corporal en general. En referencia al deporte, Alarcón, Ureña, Castillo, Martín y Cárdenas (2017) sostienen que los jugadores que se destacan son aquellos que poseen la habilidad para generar un elevado nivel de incertidumbre en el rival al dar una respuesta novedosa ante una situación de juego ya conocida. Es en este sentido que entra en juego la creatividad como capacidad de afrontar los obstáculos que se van presentando en el juego, ser capaces de predecir las acciones de los adversarios y para establecer conductas de anticipación. La danza también es una actividad propicia para la creatividad tal como hemos observado en estudios anteriores con integrantes de un taller folclórico. Crear coreografías, integrar movimientos, sonidos y expresiones son actividades que promueven el pensamiento divergente y la construcción de productos originales en la danza (Arbillaga, Elisondo y Melgar, 2018).

En el deporte... porque me gusta cómo me sale esa actividad... (Clara, 31 años).

Cuando más creativa soy es cuando bailo, mi mente se relaja. En cada paso intento ser creativa y ponerle mi estilo (María Luz, 20 años).

Observamos que las personas manifiestan ser creativas en diferentes acciones y situaciones

\section{Diólo oos}


de la vida diaria. En el grupo estudiado, la creatividad se asocia con diferentes campos de acción, como cocinar, manualidades, actividades académicas, diseñar y resolver problemas cotidianos. Las expresiones de los participantes están vinculadas a las consideraciones de Richards (2010) sobre la creatividad cotidiana como una manifestación de originalidad en el trabajo y el tiempo libre. Como sostiene la autora, la creatividad no se limita a las áreas artísticas, sino que abarca todas las actividades de la vida diaria. Los participantes señalan diferentes dominios creativos, estas consideraciones se vinculan con los planteos que reconocen las particularidades de los procesos creativos en diversos campos (Baer, 2015; Kaufman, 2012).

Además de las áreas artísticas, laborales y académicas, se destaca en las expresiones de los participantes actividades creativas orientadas a la resolución de problemas cotidianos y la generación de vínculos cooperativos con otras personas. La vida cotidiana, las relaciones interpersonales, el cuidado de otras personas y el afrontamiento de problemas son áreas donde la creatividad también es posible, tal como sostienen los participantes y los planteos actuales sobre procesos creativos emocionales. La creatividad emocional refiere a la capacidad de las personas de conectarse con sus propias emociones y regularlas creativamente en diversas situaciones y problemas. Es un patrón de habilidades cognitivas y rasgos de personalidad relacionados con la originalidad y la adecuación en la experiencia emocional (Ivcevic, Brackett y Mayer, 2007).

\section{Emociones en el proceso creativo}

Interesa en esta categoría analizar emociones que los participantes asocian con los procesos creativos. En la mayoría de los casos, observamos emociones positivas, sensaciones de placer y bienestar durante el desarrollo de acciones creativas. Sin embargo, también aparecen aspectos negativos como miedo, ansiedad y frustración, especialmente en los casos en los que los productos elaborados no satisfacen a los autores.

Me dio gusto. Te desenchufas un poco de la realidad, podes crear, imaginar, fantasear, ser creativo desde lo que a uno mismo le pasa, me gustó (Marcela, 30 años).

Me encanta podés hacer las cosas de distinta manera, ser flexible en ese sentido. Me hace bien... en un espacio de equilibrio y tranquilidad personal (Alejandra, 28 años).

Me siento más que bien y es mi lugar en el mundo (Leandro, 28 años).

Me siento muy bien cuando lo hago porque me conecta conmigo y expreso mis sentimientos o pensamientos, digamos que mis aspectos internos los puedo expresar o exteriorizar, y a veces, compartirlo con otros (Gabriela, 22 años).

Feliz, contento. Me satisface poder hacer esas cosas (Alejandro, 52 años). 
Realizada, satisfecha de haber proyectado bien, o frustrada de no haber explayado bien mi idea (Agustina, 20 años).

Las expresiones de los participantes se relacionan con estudios anteriores que indican los impactos de las actividades creativas en el bienestar general de las personas (Richard, 2010) y el desarrollo de emociones positivas (Benedek, Jauk, Kerschenbauer, Anderwald y Grond, 2017; Conner, DeYoung y Silvia, 2018; Karwowski, Lebuda, Szumski y Firkowska-Mankiewicz, 2017; Silvia et al, 2014). Los participantes usan palabras como felicidad, placer, alivio, relajación y satisfacción para definir cómo se sienten durante y después de las actividades creativas. Estas actividades también constituyen espacios para la expresión personal, la autorrealización, el crecimiento y el desarrollo de proyectos futuros.

El despliegue de procesos creativos en contextos cotidianos parece ser un promotor de la salud en un sentido amplio, ya que fomenta las emociones positivas relacionadas con la expresión personal, la autorregulación y la autorrealización. Los estudios previos de creatividad cotidiana (Gandolfo y Marty, 2010; McCabe, 2015; Pollanen, 2015) también señalan los efectos positivos de las actividades creativas en los procesos de autoconfianza, autoestima y construcción de identidad. Del mismo modo, el desarrollo de procesos creativos promueve la autonomía, el desarrollo personal y la comunicación con otras personas.

Pertenencia e identidad se interrelacionan en las voces de los participantes cuando se refieren al desarrollo de actividades creativas. En este sentido, acordamos con Glaveanu (2017), en la necesidad de investigar el Ser creativo y los elementos que lo constituyen. El análisis de las creencias construidas por las personas con respecto a sus propias habilidades creativas es relevante para comprender los procesos de despliegue de la creatividad en contextos cotidianos. La identidad se construye en la compleja red de representaciones con respecto a las capacidades y los logros propios, integrando experiencias pasadas, actividades presentes y proyectos futuros. La identidad creatividad se construye y reconstruye a partir de interacciones entre el Yo y los otros, en determinado contexto sociocultural.

\section{Creatividad en contextos educativos}

En esta categoría nos interesa analizar percepciones de los participantes respecto de factores que condicionan el despliegue de la creatividad en contextos educativos. Los entrevistados consideran que la creatividad es una herramienta indispensable en los contextos educativos para la construcción de aprendizajes significativos. Estas expresiones se vinculan con los planteos de Novak (2013) respecto de las relaciones entre creatividad y aprendizaje significativo, y también con los referidos a la minicreatividad (comprensiones novedosas respecto de los conocimientos) como proceso inherente a los aprendizajes que permiten generar (Kaufman y Be-

Diólopos sobre Educación año 11 | número 20 | enero-junio 2020 | ISSN 2007-2171 
ghetto, 2009).

...el día que una persona encargada de educar pierda creatividad es el momento en que debe dedicarse a otra cosa, sino todo lo que transmita va a comenzar a ser una mera repetición de la teoría, perdiendo así todo tipo de significatividad los aprendizajes y experiencias transmitidas. Un educador debe estar siempre predispuesto y abierto totalmente a la creatividad y a la innovación para no caer en la monotonía en su tarea de enseñar y aprender (Aldana, 21 años).

En concordancia con teorías actuales de los contextos educativos creativos (Davies et al, 2013; Gajda, Beghetto y Karwowski, 2017; Glaveanu, 2018; Richardson y Mishra 2018), los entrevistados señalan la importancia en la creatividad del aprendizaje experiencial y de las actividades que demandan la resolución de problemas reales y la generación de productos alternativos.

...siempre me acuerdo de una profe de historia del secundario que nos hacía teatralizar el tema que iba dando, eso me ayudó a no olvidarme jamás... (Valentina, 34 años).

Es imprescindible, porque debemos pensar que con creatividad se pueden resolver distintos problemas, tenemos que intentar de que las respuestas sean múltiples y los caminos para llegar a esas respuestas sean variados, y ahí el docente tiene que pensar antes esos caminos que el niño o el adulto tiene que recorrer para llegar a resolver, desde el, vamos, la creatividad está presente, desde el planteo de una actividad tiene que estar presente la creatividad (Viviana, 52 años).

Influye en razonar una idea para materializarla, contextualizarla, y hacerla realidad, influye en poner en juego nuestra imaginación y desafiarnos a pensar hasta donde podemos crear e idealizar, influye a comunicarnos a expresar lo que ronda en la cabeza de uno y transmitir un mensaje y compartirlo con nuestros pares, influye saber que no todo es exacto que cada idea tiene un toque de subjetividad y que ello hace que lo que creamos es infinito y tiene muchísimas posibilidades diferentes de interpretación (Valentina, 34 años)

Los participantes también se refieren al apoyo institucional y el compromiso con proyectos innovadores como un requisito indispensable para crear contextos novedosos de enseñanza. Las creencias y representaciones de los actores institucionales juegan un papel central en los cambios educativos. Tal como señala Bolívar (1999), las innovaciones educativas suponen cambios en las prácticas que siempre se sustentan en creencias y concepciones nuevas de los protagonistas.

Para promover la creatividad, no sé si sería una característica puntual, pero, para mi es fundamental que 
los encargados del contexto educativo o directivos como quieras llamarlo promuevan y acompañen la creatividad de cada docente entendiendo que se debe buscar construir un mejor clima de enseñanzaaprendizaje poniendo al alumno como el principal protagonista del quehacer educativo. Y también para mi es súper importante contar con docentes o un equipo de trabajo que siga la misma línea de pensamiento (Aldana, 21 años).

Cualquier característica en la que la persona que asiste a la institución tenga la libertad de poder ser el, de poder tener la posibilidad de crear y recrear en todo momento, de construir y des-construir, ya sean conocimientos, aprendizajes (Giuliana, 24 años).

Asimismo, los participantes destacan el protagonismo que se les debe asignar a los alumnos en los procesos educativos y la necesidad de espacios de libertad en los que las personas puedan desarrollarse. Las expresiones de los participantes pueden vinculase con los planteos de los especialistas referidos a las innovaciones decisivas en el campo educativo:

Llamamos innovaciones decisivas a aquellas experiencias, proyectos o metodologías que pueden ser implementadas en una institución educativa para generar una poderosa apropiación del conocimiento por parte de los alumnos, movilizando la voluntad, el deseo, la pasión de aprender. Las innovaciones decisivas buscan crear comunidades de aprendizaje y postulan principios de aprendizaje dialógico (Freire, 1996), pragmatismo democrático (Dewey, 1996) y educación para la comprensión (Perkins, 2010). (Rivas et al., 2017: 9)

Los participantes también identifican algunas dificultades u obstáculos a la hora de generar contextos creativos de enseñanza y aprendizaje. Los entrevistados se refieren a condicionantes personales que obstaculizan los cambios (mentes cerradas, rechazo al cambio, falta de compromiso y capacitación) y también a cuestiones institucionales y políticas educativas (Bajadas de línea, normalización de las prácticas, autoritarismos, problemas económicos, conflictos de intereses, etc.). Craft (2003) también reconoce diversos obstáculos a la hora de generar contextos creativos de enseñanza y aprendizaje. La autora se refiere a dificultades vinculadas con el uso de terminología apropiada, a los conflictos entre las políticas educativas y las prácticas, a la organización del currículum y a los modelos pedagógicos vigentes.

Yo creo que pueden influenciar negativamente lo que te dije por ahí, un poco la falta de libertad, la monotonía, las clases siempre igual, el sistema educativo es un sistema ambiguo digamos y contradictorio porque es un sistema, en donde hay una serie de pautas, una jerarquía y todo eso lleva a que por ahí como que no tenga lugar la creatividad (...) lo económico puede también influenciar negativamente, aunque no del todo, siempre hay cosas que se pueden hacer y no hace falta dinero. (...) la falta de ac-

\section{Diólo@os}


tualización me parece de los docentes, que no se capaciten, que no busquen seguir estudiando, que se queden como durmiéndose en los laureles (Luis, 34 años)

¿Factores negativos? Muchos, ya con el solo hecho de esa mirada antigua, esa normatividad de que se tienen que sentar de a dos, tienen que hacer silencio en el momento que el docente les da actividades para hacer. Otro factor negativo claramente es que el docente espere la misma respuesta a un problema, que si no lo resuelve de la manera que el profesor espera está mal (Giuliana, 24 años)

Creo que los factores negativos, son las mismas personas que no quieren que se produzcan cambios creativos dentro de la educación (Alejandra, 33 años).

Creo que lo que más influye negativamente dentro del ámbito de la educación a nivel de la creatividad es el hecho de que existan a veces mentes cerradas y conservadoras que no permitan a veces, que se desarrolle prácticas educativas distintas a las tradicionales (Bárbara, 26 años).

Los entrevistados han identificado condiciones que promueven u obstaculizan el desarrollo de la creatividad en contextos educativos. En concordancia con los planteos de especialistas en la temática, los entrevistados vinculan la creatividad en contextos educativos con el aprendizaje experiencial, la libertad, la autonomía, la búsqueda de alternativas y la resolución de problemas diversos. Según las expresiones de los participantes, los docentes juegan un papel importante en la construcción de contextos creativos para la enseñanza y el aprendizaje. En esta línea, señalan la necesidad de formación y actualización de los profesores. Asimismo, destacan el rol de las instituciones y sus autoridades en el acompañamiento de procesos creativos e innovadores. En suma, los contextos educativos creativos parecen ser posibles solo si las iniciativas personales de docentes y alumnos son andamiadas por las instituciones y las políticas educativas. También destacamos la incidencia de las concepciones construidas por los actores respecto de la creatividad y las posibilidades de promoverla en contextos educativos. Los cambios y las innovaciones dependen de estas teorías implícitas respecto del aprendizaje, la creatividad y los contextos educativos.

\section{Consideraciones finales}

Las voces de los participantes indican que la creatividad es un potencial de todas las personas que puede desplegarse en las más diversas actividades cotidianas. Esta potencialidad está vinculada a la originalidad, la imaginación y la resolución de problemas. Las percepciones de los entrevistados se relacionan con planteos actuales en el campo de investigación de los procesos creativos, particularmente con los estudios de la creatividad cotidiana, la identidad creativa y los contextos que resultan propicios para la creación. Asimismo, los participantes se han refe- 
rido a las emociones y las relaciones interpersonales como dominios en los cuales es posible desarrollar la creatividad. También han manifestado vinculaciones entre actividades creativas, emociones positivas, placer y satisfacción personal. Desarrollar procesos creativos en la vida cotidiana parece promover el bienestar y la salud de las personas, tal como lo señalan los participantes y se evidencia en estudios anteriores.

En suma, reconocer que la creatividad es una potencialidad de todas las personas, que no solo se limita a cuestiones artísticas sino que puede desplegarse en diferentes ámbitos sociales, culturales, interpersonales y emocionales, es relevante para la construcción de contextos creativos de enseñanza y aprendizaje. Docentes y alumnos pueden desarrollar actividades creativas en las ciencias, las artes, las emociones y las relaciones interpersonales. Además, la creatividad no es un proceso individual sino que implica, de manera directa o indirecta, a otras personas. En los entornos educativos también es necesario comprender el carácter social de la creatividad y propiciar tareas colaborativas de construcción de conocimientos alternativos.

Para cimentar contextos educativos creativos es indispensable desarrollar concepciones amplias de la creatividad, porque extensas son las potencialidades de las personas y los campos donde la creatividad es posible. También es necesario ampliar las posibilidades educativas, no poner límites al pensamiento y la acción, asumiendo los riesgos necesarios y los desafíos que implica crear nuevos conocimientos.

Si la creatividad nos hace felices, promover acciones creativas en contextos educativos parece ser una interesante herramienta contra la monotonía, el aburrimiento y el desgano de docentes y estudiantes. En las instituciones educativas también podemos ser creativos con nuestras emociones y relaciones interpersonales, buscando formas alternativas de manejar situaciones y vínculos con otras personas.

Crear contextos creativos implica desafíos, compromisos y riesgos para los docentes y las instituciones. El trabajo colaborativo entre profesores parece ser un camino propicio para sostener los procesos innovadores y enfrentar los obstáculos que se presentan al intentar generar cambios en los espacios educativos. También son necesarios compromisos de las autoridades institucionales y aperturas en las políticas educativas que generen intersticios donde las innovaciones sean posibles.

En definitiva, la creatividad como proceso complejo incluye componentes subjetivos y contextuales, requiere de ciertas condiciones cognitivas, personales, sociales y culturales para desplegarse en diferentes áreas de conocimiento. Reconocer a la creatividad como proceso complejo y potencialidad de las personas es una contribución importante para el campo educativo y la búsqueda de espacios innovadores de enseñanza y aprendizaje. Con el presente estudio hemos procurado mostrar percepciones sobre creatividad de un grupo de personas que, a nuestro criterio, contribuyen a generar nuevas miradas y perspectivas respecto del aprendizaje y la enseñanza creativa. Las percepciones de los participantes ponen en tela de juicio imágenes 
y estereotipos que asocian a la creatividad solo con algunos campos de conocimiento y que la consideran exclusividad de unas pocas personas. Pensar en contextos educativos creativos solo tiene sentido si reconocemos las potencialidades de las personas y las diferentes áreas donde es posible innovar.

Interesantes líneas de investigación emergen del presente estudio como, por ejemplo, analizar en profundidad significados y sentidos construidos por docentes respecto de sus prácticas educativas innovadoras. También es relevante en próximos estudios recuperar las voces de los alumnos identificando potencialidades y desempeños creativos en contextos educativos. Las perspectivas de directivos y de los responsables de las políticas educativas respecto de la creatividad y el lugar que esta ocupa en los lineamientos curriculares, también es una temática de relevancia para otras investigaciones. Asimismo, como futura línea de indagación se proponen estudios que incluyan grupos de personas que se destacan por sus despeños creativos en diferentes campos artísticos, culturales y científicos.

Las escuelas no siempre matan la creatividad, frecuentemente ofrecen espacios para que florezca...

\section{Referencias bibliográficas}

Alarcón, F., N. Ureña, A. Castillo, D. Martín y D. Cárdenas (2017). Las funciones ejecutivas como predictoras del nivel de pericia en jugadores de baloncesto. Revista de psicología del deporte, 26(1), 71-74.

Arbillaga, L., Elisondo, R.C. \& Melgar, M.F. (2018). Espacios que proyectan hacia nuevos horizontes. La danza como puente entre la creatividad y lo cotidiano. Estudios sobre Arte Actual, 6, 21-33.

Arizpe, L. (2001). Cultura, creatividad y gobernabilidad. En D. Mato (comp.). Estudios Latinoamericanos sobre cultura y transformaciones sociales en tiempos de globalización. Buenos Aires: CLACSO. http://biblioteca.clacso.edu.ar/clacso/gt/20100912034428/estudios.pdf

Baer, J. (2015). Domain Specificity of Creativity. Oxford: Academic Press.

Beghetto, R. y M. Karwowski (2017). Toward Untangling Creative Self-Beliefs. En M. Karwowski y J.C. Kaufman (eds.). The Creative Self: Effect of Beliefs, Self-efficacy, Mindset, and Identity. Oxford: Academic Press, 3-22.

(2017). Creativity in Teaching. En: J.C. Kaufman, J. Baer y V.P. Glăveanu (eds.). Cambridge Handbook of Creativity Across Different Domains. Nueva York: Cambridge University Press.

Benedek, M., R. Bruckdorfer y E. Jauk (2019). Motives for Creativity: Exploring the What and Why of Everyday Creativity. The Journal of Creative Behavior. https://onlinelibrary.wiley.com/doi/ full/10.1002/jocb.396 
,E. Jauk, K. Kerschenbauer, R. Anderwald y L. Grond (2017). Creating Art: An Experience Sampling Study in the Domain of Moving Image Art. Psychology of Aesthetics, Creativity, and the Arts, 11(3), 325-334. http://dx.doi.org/10.1037/aca0000102

Bolívar, A. (1999). Cómo mejorar los centros educativos. Madrid: Síntesis.

Conner, T.S., C.G. DeYoung y P.J. Silvia (2018). Everyday Creative Activity as a Path to Flourishing. The Journal of Positive Psychology, 13(2), 181-189.

Corbalán Berná, J. (2008). ¿De qué se habla cuando hablamos de creatividad? Cuadernos de la Facultad de Humanidades y Ciencias Sociales, 35, 11-21. https://www.redalyc.org/ html/185/18512511002/

Creswell, J.W. y Poth, C.N. (2007). Qualitative Inquiry and Research Design: Choosing among Five Approaches. Londres: Sage publications.

Craft, A. (2003). The Limits to Creativity in Education: Dilemmas for the Educator. British Journal of Educational Studies, 51(2), 113-127.

Davies, D., D. Jindal-Snape, C. Collier, R. Digby, P. Hay y A. Howe (2013). Creative Learning Environments in Education. A Systematic Literature Review. Thinking Skills and Creativity, 8 , 80-91.

Elisondo, R. (2016). Creativity is Always a Social Process. Creativity. Theories-Research-Applications, 3(2), 194-210.

Gajda, A., R. Beghetto y M. Karwowski (2017). Exploring Creative Learning in the Classroom: A Multi-method Approach. Thinking Skills and Creativity, 24, 250-267.

Gandolfo, E. y G. Marty (2010). Women Doing it Forever: the Everyday Creativity of Women Craftmakers. Australian and New Zealand Journal of Art Therapy, 5(1), $29-43$.

Glaveanu, V. (2013). Rewriting the Language of Creativity: The Five A's Framework. Review of General Psychology, 17, 69-81. doi: 10.1037/a0029528

(2014). The Psychology of Creativity: A Critical Reading. Creativity: Theories-Research-Applications, 1, 10-32. doi: 10.15290/ctra.2014.01.01.02

(2017) The Creative Self in Dialogue. En: M. Karwowski y J.C. Kaufman (eds.). The Creative Self: Effect of Beliefs, Self-efficacy, Mindset, and Identity. Oxford: Academic Press, 117-135.

(2018) Educating which creativity? Thinking Skills and Creativity, 27, 25-32.

Helfand, M., J.C. Kaufman y R. Beghetto (2016). The Four-C Model of Creativity: Culture and Context. En: The Palgrave Handbook of Creativity and Culture Research Reino Unido: Palgrave Macmillan.

Ivcevic, Z., M.A. Brackett y J.D. Mayer (2007). Emotional Intelligence and Emotional Creativity. Journal of personality, 75(2), 199-236.

Karwowski, M., I. Lebuda, G. Szumski y A. Firkowska-Mankiewicz (2017). From Moment-to-Moment to Day-to-Day: Experience Sampling and Diary Investigations in Adults' Everyday 
Creativity. Psychology of Aesthetics, Creativity, and the Arts, 11(3), 309-324. http://dx.doi. org/10.1037/aca0000127

Kaufman, J. (2012). Counting the Muses: Development of the Kaufman Domains of Creativity Scale (K-DOCS). Psychology of Aesthetics, Creativity, and the Arts, 6, 298-308. DOI: 10.1037/ $\underline{\mathrm{t} 17613-000}$

y R. Beghetto (2009). Beyond Big and Little: The Four C Model of Creativity. Review of General Psychology, 13(1), 1-12.

R. Beghetto y C. Watson (2016). Creative Metacognition and Self-ratings of Creative Performance: A 4-C Perspective. Learning and Individual Differences, 51, 394-399. DOI: org/10.1016/j. lindif.2015.05.004

Kettler, T., K. Lamb, A. Willerson y D.R. Mullet (2018). Teachers' Perceptions of Creativity in the Classroom. Creativity Research Journal, 30(2), 164-171, DOI: 10.1080/10400419.2018.1446503

Kozbelt, A., R. Begheto y M. Runco (2010). Theories of Creativity. En: J. Kaufman y R. Sternberg (eds.). The Cambridge Handbook of Creativity. Cambridge: Cambridge Press, 447-463.

McCabe, M., y T. de Waal Malefyt (2015). Creativity and Cooking: Motherhood, Agency and Social Change in Everyday Life. Journal of Consumer Culture, 15(1), 48-65.

Mullet, D., A. Willerson, K. Lamb y L. Kettler (2016) Examining Teacher Perceptions of Creativity: A Systematic Review of the Literature. Thinking Skills and Creativity, 21, 9-30. https://doi. org/10.1016/j.tsc.2016.05.001.

Novak, J. (2013). Meaningful Learning is the Foundation for Creativity. Revista Qurriculum, 26, 27-38.

Pollanen, S. (2015). Elements of Crafts that Enhance Well-being: Textile Craft Makers' Descriptions of their Leisure Activity. Journal of Leisure Research, 47(1), 58-78.

Pretz, J. y J. Kaufman (2015). Do Traditional Admissions Criteria Reflect Applicant Creativity? The Journal of Creative Behavior, 51(3), 240-251.

Richards, R. (2010). Everyday Creativity. En: J. Kaufman y R. Sternberg (eds.). The Cambridge handbook of Creativity. Cambridge: Cambridge University Press, 189-215.

Richardson, C. y P. Mishra (2018). Learning Environments that Support Student Creativity: Developing the SCALE. Thinking Skills and Creativity, 27, 45-54.

Rivas, A., F.André, L.Delgado, I.Aguerrondo, R.Anijovich,M.Furmany A.Vota(2017).50innovaciones educativas para escuelas. Lima: Centro de Implementación de Políticas Públicas para la Equidad y el Crecimiento. http://disde.minedu.gob.pe/bitstream/handle/MINEDU/5587/50\%20 innovaciones\%20educativas\%20para\%20escuelas.pdf?sequence $=1$ \&isAllowed $=y$

Runco, M.A. (2019). Creativity as a Dynamic, Personal, Parsimonious Process. En R. Beghetto y G. Corazza (eds.). Dynamic Perspectives on Creativity. Cham: Springer, 181-188.

Runco, M.A. y G.J. Jaeger (2012). The Standard Definition of Creativity. Creativity Research Journal, 24(1), 92-96. 
Said-Metwaly, S., W. Van den Noortgate y E. Kyndt (2017). Methodological Issues in Measuring Creativity: A Systematic Literature Review. Creativity. Theories - Research - Applications, 4(2), 276-301. DOI: 10.1515/ctra-2017-0014

Silvia, P.J., R.E. Beaty, E.C. Nusbaum, K.M. Eddington, H. Levin-Aspenson y T.R. Kwapil (2014). Everyday Creativity in Daily Life: An Experience-sampling Study of "Little C"Creativity. Psychology of Aesthetics, Creativity, and the Arts, 8(2), 183-188. DOI: 10.1037/a0035722

Soneira, A. (2006). La teoría fundamentada en los datos (grounded theory) de Glaser y Strauss. En Vasilachis, I. Estrategias de investigación cualitativa. (pp. 153-173). Barcelona: Gedisa.

Trnka, R., M. Zahradnik y M. Kuška (2016). Emotional Creativity and Real-Life Involvement in Different Types of Creative Leisure Activities. Creativity Research Journal, 28(3), 348-356.

Türst, G. y F. Grin (2018). A Comprehensive Method for the Measurement of Everyday Creativity. Thinking Skills and Creativity, 28, 84-97. https://doi.org/10.1016/j.tsc.2018.03.007

UNESCO (1994). Nuestra diversidad creativa. https://unesdoc.unesco.org/ark:/48223/ pf0000105586 spa

(2014). Gender Equality, Heritage and Creativity. https://unesdoc.unesco.org/ark:/48223/ pf0000229418

(2015) Cultural Times The first Global Map of Cultural and Creative Industries https:// en.unesco.org/creativity/sites/creativity/files/cultural times. the first global map of cultural and creative industries.pdf Accessed 20 December 2018

Ward, T.B. (2007). Creative Cognition as a Window on Creativity. Methods, 42(1), 28-37. 